\title{
2-Oxiranecarbonitriles in the synthesis of linked quinono heterocyclic derivatives
}

\author{
Julija Volmajer, ${ }^{a}$ Renata Toplak, ${ }^{* a}$ Shmuel Bittner, ${ }^{b}$ and Alenka Majcen Le Marechal ${ }^{\mathrm{a}}$ \\ ${ }^{a}$ University of Maribor, Faculty of Mechanical Engineering, Smetanova 17, \\ 2000 Maribor, Slovenia and ${ }^{b}$ Ben-Gurion University of Negev, Department of Chemistry, \\ P.O.Box 653, Beer Sheva 84105, Israel \\ E-mail: renata.toplak@uni-mb.si
}

\section{Dedicated to Professor Branko Stanovnik, University of Ljubljana, on his $65^{\text {th }}$ birthday (received 08 Aug 03; accepted 18 Sep 03; published on the web 22 Sep 03)}

\begin{abstract}
Alkyl protected equivalents of hydroquinono-2-oxiranecarbonitriles $\mathbf{3}$ were prepared and applied in the synthesis of thiazolinone 5 and 7, thiazole 8 and oxathiole $\mathbf{9}$ derivatives directly linked to the precursor of the quinonic part of the molecule. The synthesis of 2-[(1,4naphthoquinolyl)amino]thiazole derivatives $\mathbf{1 1}$ is also reported.
\end{abstract}

Keywords: Oxirane, quinone, thiazole, oxathiole

\section{Introduction}

The importance of 2,2-dicyanooxiranes and 2-cyano-2-alkoxycarbonyloxiranes as intermediates in organic synthesis has been extensively investigated. ${ }^{1}$ Because of their multifunctional structure they proved to be versatile reagents in the synthesis of a large variety of carbocyclic, heterocyclic or acyclic derivatives. In general, nucleophilic reagents can react with 2cyanooxiranes regioselectively: they can attack the oxirane ring, cyano or alkoxycarbonyl functional group to give either ring opened products, new functionalised oxiranes or different heterocycles. Ring opening can be achieved with a variety of heteroatomic nucleophiles and their attack is followed by hydrogen cyanide elimination to give through non stable cyanohydrine and cyanoformyl intermediates among others also $\alpha$-ketoesters or $\alpha$-ketoamides (pyruvamides) ${ }^{2,3}$. In acidic conditions with halohydric acids $\alpha$-haloketone analogues are formed. The bielectrophilic nature of 2-cyanooxiranes and their $\alpha$-haloketone derivatives enables formation of a variety of heterocyclic compounds of pharmaceutical interest such as thiazoles, ${ }^{4,5}$ dithioles, ${ }^{6}$ imidazoles, ${ }^{7}$ 1,3-oxathioles, ${ }^{8} 1,3$-oxaselenoles ${ }^{9}$ and condensed imidazolo and thiazolo derivatives. ${ }^{10}$ 
On the other hand, quinonic compounds are ubiquitous in nature ${ }^{11}$ and implicated in numerous cellular functions involving mechanisms of electron and hydrogen transfers. ${ }^{12}$ Some natural or synthetic quinonic derivatives are widely used as drugs for treatment of human cancer $^{13,14}$ or antibacterial drugs ${ }^{15}$, they also exhibit antimalarial ${ }^{16}$ and antifungal ${ }^{17}$ activity. Aminoquinones represent as well an important group of biologically active compounds involved in enzyme inhibition, DNA cross-linking, antibacterial, antifungal and anticancer activity. ${ }^{18}$ The need for a variety of quinonic compounds and libraries of such materials for different biological testing prompted us to plan the synthesis of multifunctional starting material from which a large variety of quinonic derivatives might be prepared.

In this publication we present the synthesis of several alkyl protected equivalents of hydroquinono-2-oxiranecarbonitrile and their further transformation into heterocyclic derivatives directly linked to the precursor of the quinonic part of the molecule. The synthesis of aminoquinonic derivatives is also reported.

\section{Results and Discussion}

2-Oxiranecarbonitrile reagents 3 were prepared in a two-step procedure outlined in Scheme 1. We chose starting 2,5-dimethoxy- or 3,4,5-trimethoxy-benzaldehyde $\mathbf{1}$ as alkyl protected equivalent of quinonic part of the molecule. According to the Knoevenagel procedure ${ }^{19}$ it reacted with malononitrile derivatives in the presence of catalytic amount of piperidine. Styryl derivatives 2 were formed in high yields after condensation at room temperature. ${ }^{1} \mathrm{H}$ NMR analysis of a crude product obtained in the reaction between 2,5-dimethoxybenzaldehyde 1a and ethyl cyanoacetate revealed formation of only one geometric isomer, according to the crystal structure determination, ${ }^{20} E$-styryl isomer 2c. Our result is in accordance with literature data. ${ }^{19}$

In the second step alkenes 2 were treated with an aqueous solution of sodium hypochlorite causing oxidation of the double bond and yielding oxiranes 3 . Due to electron donating methoxy substituents on the phenyl ring reaction is highly $\mathrm{pH}$ sensitive. With variation of $\mathrm{pH}$ of the reaction medium and concentration of sodium hypochlorite solution two different products were obtained from 2-[(2,5-dimethoxyphenyl)methylen]malononitrile 2a. Selective formation of oxirane ring proceeded in a reaction medium with $\mathrm{pH} 8-9$ (compounds $3 \mathbf{a}, 3 \mathbf{3}$ ) and 3\% solution of sodium hypochloride. This is in contrast to the previously published synthesis of 3-aryl substituted oxiranes with electron withdrawing groups attached on the phenyl ring, needing acidic medium for their formation. ${ }^{21,22}$ In acidic medium oxidation of the double bond was accompanied with electrophilic aromatic chlorination yielding compund 3c. Three methoxy substituents facilitate accompanying electrophilic aromatic chlorination even in basic medium $(\mathrm{pH}>9)$. 


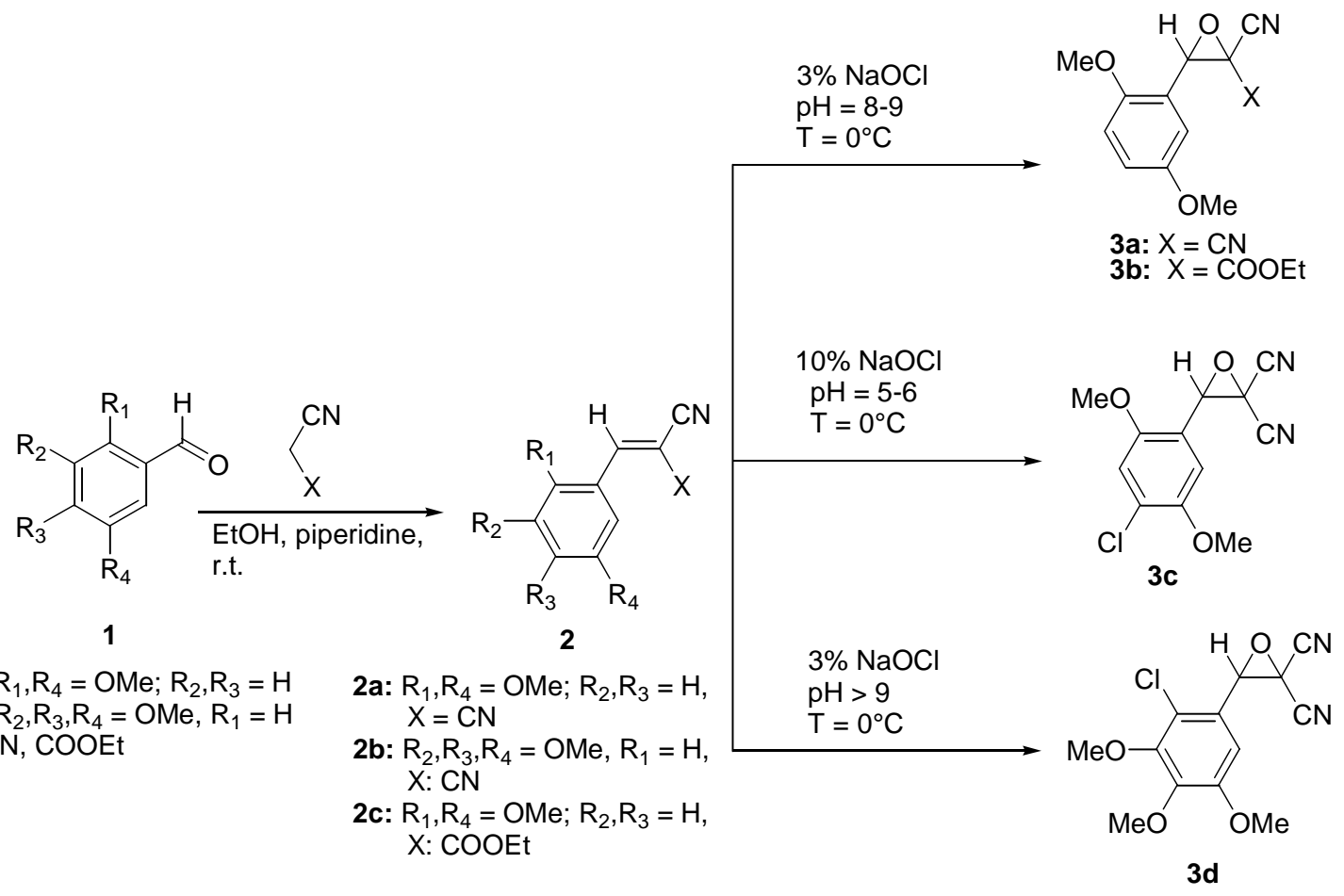

\section{Scheme 1}

2-Cyanooxiranes 3 were further applied in the preparation of the heterocyclic compounds directly linked to the precursor of the qouinonic part of the molecule. In the reactions with thiourea derivatives presented in Scheme 2 and 3 the bielectrophilic nature of cyanooxiranes was exploited.

Simple stirring of 2,2-dicyanooxirane reagents $3 \mathbf{a}$ and $\mathbf{3 d}$ with thioureas $\mathbf{4 a}, \mathbf{4} \mathbf{b}$ and $\mathbf{4 c}$ in acetonitrile at room temperature gave thiazolinone derivatives 5 in yields ranging between $36 \%$ and $70 \%$ for purified products. The structure of products was determined by IR, ${ }^{1} \mathrm{H} \mathrm{NMR},{ }^{13} \mathrm{C}$ NMR, mass spectra and elemental analysis and comparison with previously published results. ${ }^{5}$ ${ }^{1} \mathrm{H}$ NMR and ${ }^{13} \mathrm{C}$ NMR spectra of thiazolinones 5a-d in the solution of DMSO- $d_{6}$ revealed only one set of signals suggesting existence of only one tautomeric form under this condition. A peak in ${ }^{1} \mathrm{H}$ NMR appearing as singlet at $\delta$ between 5.3-6.0 ppm for the cyclic $\mathrm{sp}^{3} \mathrm{CH}$ structural element and a signal at $\delta$ 172.7-181.0 ppm for carbonyl group in ${ }^{13} \mathrm{C}$ NMR suggest existence of thiazolinone tautomeric form $\mathbf{5 A}$ in $\mathrm{DMSO}-d_{6}$ solution. The $\mathrm{C}=\mathrm{O}$ absorption band observed between $1680-1720 \mathrm{~cm}^{-1}$ in IR (KBr) spectra indicates the same tautomeric form $\mathbf{5 A}$ also in the solid state. Structure of the compound 5a was confirmed by X-ray analysis determined 2aminothiazolinone form in the solid state (Figure 1). ${ }^{23}$ On the contrary, ${ }^{1} \mathrm{H}$ NMR and ${ }^{13} \mathrm{C}$ NMR spectra for the compounds $\mathbf{5 e}$ and $\mathbf{5 f}$, in solution in DMSO- $d_{6}$ indicate equilibrium between thiazolinone 5A and 4-hydroxythiazole 5B tautomeric forms. In the ${ }^{1} \mathrm{H}$ NMR spectra of both compounds two sets of signals were observed for all the protons exept for cyclic $\mathrm{CH}$ structural element at the position 5 which appears as a singlet at $\delta 5.3-5.5 \mathrm{ppm}$ and $\mathrm{OH}$ at $\delta 11.7-11.9 \mathrm{ppm}$. The ratio between tautomeric forms $\mathbf{5 A}$ and $\mathbf{5 B}$ in the compound $5 \mathbf{e}$ is $1.3: 1$ and $5 \mathbf{f} 1.7: 1$. 
Treatment of 2,2-dicyanooxirane 3a with thiohydantoin in acetonitrile at room temperature yielded imidazo[2,1-b]thiazol-3,6-dione derivative 7. This results from heterocyclisation of the more nucleophilic nitrogen atom with cyanoformyl intermediate $6 .{ }^{1} \mathrm{H} \mathrm{NMR}$ and ${ }^{13} \mathrm{C} \mathrm{NMR}$ spectral analysis of the product is in good agreement with literature data., 24<smiles>[R]c1cc(C2OC2(C#N)C#N)c([R])c([R])c1[R]</smiles>

3a: $R_{1}, R_{4}=$ OMe; $R_{2}, R_{3}=H$,

3d: $R_{2}, R_{3}, R_{4}=O M e, R_{1}=C l$,

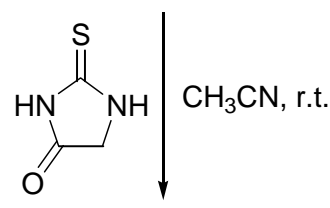<smiles>COc1ccc(OC)c(C(SC2=NC(=O)CN2)C(=O)C#N)c1</smiles>

6

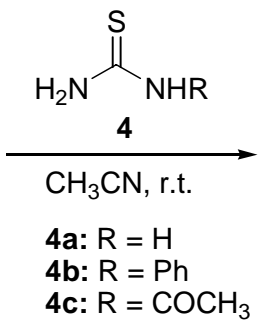

4c: $\mathrm{R}=\mathrm{COCH}_{3}$<smiles>[R]NC1=NC(=O)C(c2cc([R4])c([R3])c([R2])c2[R1])S1</smiles>

5a: $\mathrm{R}_{1}, \mathrm{R}_{4}=\mathrm{OMe} ; \mathrm{R}_{2}, \mathrm{R}_{3}=\mathrm{H}, \mathrm{R}=\mathrm{H}$ 5b: $R_{2}, R_{3}, R_{4}=O M e, R_{1}=C l, R=H$ 5c: $\mathrm{R}_{1}, \mathrm{R}_{4}=\mathrm{OMe} ; \mathrm{R}_{2}, \mathrm{R}_{3}=\mathrm{H}, \mathrm{R}=\mathrm{Ph}$ 5d: $R_{2}, R_{3}, R_{4}=O M e, R_{1}=C l, R=P h$ 5e: $R_{1}, R_{4}=$ OMe; $R_{2}, R_{3}=H, R=C_{3} C_{3}$ 5f: $\mathrm{R}_{2}, \mathrm{R}_{3}, \mathrm{R}_{4}=\mathrm{OMe}, \mathrm{R}_{1}=\mathrm{Cl}, \mathrm{R}=\mathrm{COCH}_{3}$

\section{Scheme 2}

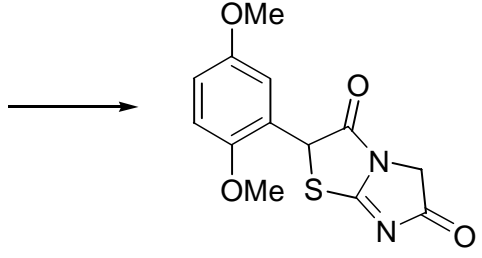

7

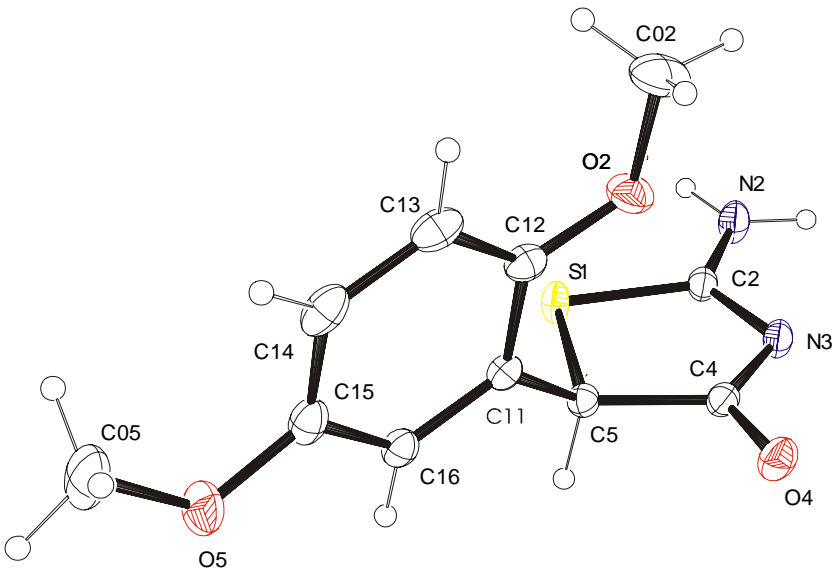

Figure 1. A view of the molecule of 5a, showing the atom numbering scheme. 
2-Cyano-2-oxiranecarboxylate $\mathbf{3 b}$ is less reactive than corresponding 2,2-dicyanooxirane analogues. Reaction between compound $\mathbf{3 b}$ and thiourea $\mathbf{4 a}$ proceeded at reflux in acetonitrile and afforded 2-aminothiazole 8 in $49 \%$ yield. The structure of the product was confirmed by Xray diffraction study. ${ }^{23}$
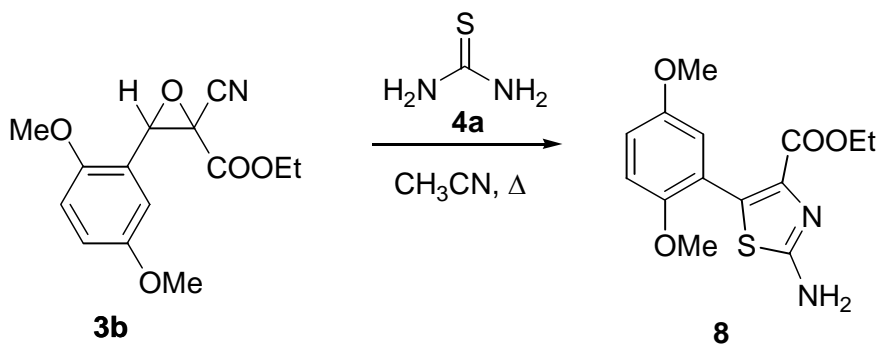

\section{Scheme 3}

Investigating the synthesis of new quinono-heterocyclic systems we used also synthetic strategy described by Breslow et al. for the preparation of 1,3-oxathiole derivatives. ${ }^{25} \mathrm{We}$ performed a reaction between 2,2-oxiranedicarbonitrile reagents (3a, 3d) and potassium thiocyanate in acetic anhydride at room temperature and isolated 2-acetylimino-1,3-oxathiole derivatives 9 as confirmed by spectroscopic methods and C, H, N microanalysis (Scheme 4). The reaction presumably proceeds through thiocyanate attack on position 3, ring-opening to cyanhydrin intermediate $\mathbf{A}$, formation of oxathiolane intermediate $\mathbf{B}$ which is finally trapped by $\mathrm{Ac}_{2} \mathrm{O}$ to yield 1,3-oxathioles $\mathbf{9}$. $^{8}$

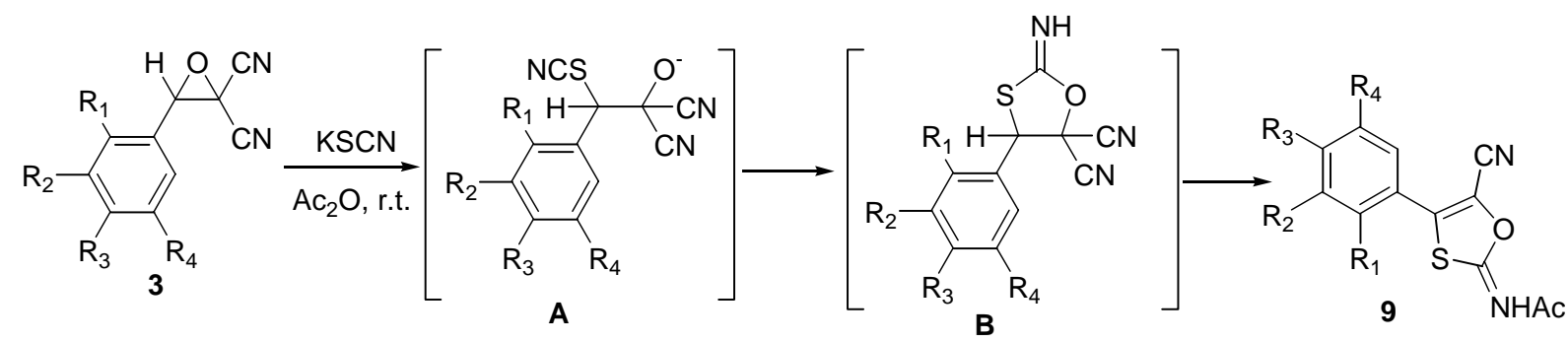

3a, 9a: $R_{1}, R_{4}=O M e ; R_{2}, R_{3}=H$,

3d, 9b: $\mathrm{R}_{2}, \mathrm{R}_{3}, \mathrm{R}_{4}=\mathrm{OMe}, \mathrm{R}_{1}=\mathrm{Cl}$,

\section{Scheme 4}

The prepared di- and trimethoxyphenyl heterocyclic systems 5, 7, 8 and $\mathbf{9}$ are now the subject of our further study focusing on the development of a method for the efficient dealkylation and transformation into connected quinono-heterocyclic systems.

As aminoquinonic compounds too present considerable interest ${ }^{18}$ we studied also the synthesis of heterocyclic compounds connected to quinono subsistent through an amino spacer 
group. Aminothiazole derivatives 5a and 8 were treated with 2,3-dichloro-1,4-naphthoquinone 10 in solution in DMF and presence of one equivalent of potassium carbonate (Scheme 5). Reaction resulted in the substitution of one chlorine atom and afforded 2-[(3-chloro-1,4naphthoquinolyl)amino]-1,3-thiazoles 11 in reasonable yields. It is interesting to note that the compound 11b undergoes a dramatic change of color with a change of the $\mathrm{pH}$ of medium. While it exhibits red color in the acidic medium it changes to deep blue color in the basic medium. This is probably due to formation of a highly conjugated resonance-stabilized anionic form $\mathbf{1 2 B} .^{26,27}$ In the case of compound 11a such phenomena were not observed.<smiles>O=C1C(Cl)=C(Cl)C(=O)c2ccccc21</smiles><smiles>CCOC(=O)c1nc(N)[se]c1-c1c(OC)cc(OC)cc1OC(C)=O</smiles>

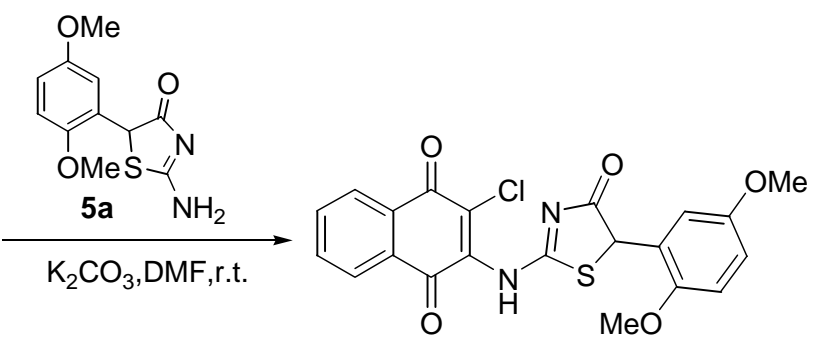

$11 \mathrm{a}$<smiles>CCOC(=O)c1nc(NC2=C(Cl)C(=O)c3ccccc3C2=O)sc1NC1=C(Cl)c2ccc(OC)cc2C1=O</smiles>

\section{Scheme 5}

In conclusion, we developed a simple synthetic approach towards linked quinonoheterocyclic systems. We prepared a series of alkyl protected equivalents of hydroquinono-2oxiranecarbonitriles which proved to be versatile reagents for the synthesis of thiazoline, thiazole and oxathiole derivatives. Transformation of di- and trimethoxyphenyl substituted heterocycles systems into connected quinono-heterocyclic systems is under investigation. As extension of our study we also prepared naphthoquinolylamino-1,3-thiazole derivatives where heterocyclic core is connected to quinono subsistent through an amino spacer group.

\section{Experimental Section}

General Procedures. Melting points were determined with Kofler hot stage apparatus. The ${ }^{1} \mathrm{H}$ NMR spectra were recorded on a Bruker Avance DPX 300 (300 MHz) spectrometer with $\mathrm{CDCl}_{3}$ 
and DMSO- $\mathrm{d}_{6}$ as solvents and TMS as internal standard. ${ }^{13} \mathrm{C}$ NMR spectra were obtained on a Bruker AM 300 spectrometer at $75 \mathrm{MHz}$ with $\mathrm{CDCl}_{3}$ and DMSO- $\mathrm{d}_{6}$ as solvents and TMS as internal standard. Mass spectra were performed on an Autospec $Q$ spectrometer. The microanalyses for C, H and $\mathrm{N}$ were obtained on a Perkin-Elmer Analyser 2400. IR spectra were determined with Perkin-Elmer 225 or 1420 spectrometer. All starting materials were commercially available (in most cases from Fluka).

General procedure for preparation of 2-(arylmethylen)malononitrile derivatives 2 Compounds were prepared according to the modified procedure described in the literature. ${ }^{28} \mathrm{~A}$ mixture of substituted benzaldehyde $1(20 \mathrm{mmol})$ and malononitrile derivative $(20 \mathrm{mmol})$ was dissolved in $30 \mathrm{ml}$ of ethanol, 5 drops of piperidine were added. The reaction mixture was stirred at room temperature for $30 \mathrm{~min}$. The product precipitated from the reaction mixture and was collected by filtration. It was recrystallized from an appropriate solvent. The following compounds were prepared in this manner:

2-[(2,5-Dimethoxyphenyl)methylen]malononitrile (2a). Prepared from 2,5-dimethoxybenzaldehyde 1a $(3.320 \mathrm{~g}, 20 \mathrm{mmol})$ and malononitrile $(1.320 \mathrm{~g}, 20 \mathrm{mmol})$ in $90 \%$ (3.852 g) yield, mp 105-106 ${ }^{\circ} \mathrm{C}$ (literature ${ }^{27} 110{ }^{\circ} \mathrm{C}$ ) (from ethanol). IR (KBr) cm ${ }^{-1}: 2226.9(\mathrm{CN})$. NMR data: $\delta_{\mathrm{H}}\left(\mathrm{CDCl}_{3}\right) 3.82\left(3 \mathrm{H}, \mathrm{s}, \mathrm{CH}_{3}\right), 3.89\left(3 \mathrm{H}, \mathrm{s}, \mathrm{CH}_{3}\right), 6.92\left(1 \mathrm{H}, \mathrm{d}, J_{\mathrm{H} 3-\mathrm{H} 4}=9.0 \mathrm{~Hz}, \mathrm{H}-3\right), 7.25$ $\left(1 \mathrm{H}, \mathrm{dd}, J_{\mathrm{H} 3-\mathrm{H} 4}=9.0 \mathrm{~Hz}, J_{\mathrm{H} 4-\mathrm{H} 6}=3.0 \mathrm{~Hz}, \mathrm{H}-4\right), 7.73\left(1 \mathrm{H}, \mathrm{d}, J_{\mathrm{H} 4-\mathrm{H} 6}=3.0 \mathrm{~Hz}, \mathrm{H}-6\right), 8.29(1 \mathrm{H}, \mathrm{s}$, $\mathrm{CH})$.

2-[(3,4,5-Trimethoxyphenyl)methylen]malononitrile (2b). Prepared from 3,4,5-trimethoxybenzaldehyde $\mathbf{1 b}(3.920 \mathrm{~g}, 20 \mathrm{mmol})$ and malononitrile $(1.320 \mathrm{~g}, 20 \mathrm{mmol})$ in $84 \%(4.099 \mathrm{~g})$ yield, mp 146-148 ${ }^{\circ} \mathrm{C}$ (literature ${ }^{29} 147-148{ }^{\circ} \mathrm{C}$ ) (from ethanol). IR (KBr) cm$~^{-1}: 2225.6(\mathrm{CN})$. NMR data: $\delta_{\mathrm{H}}\left(\mathrm{CDCl}_{3}\right) 3.91\left(6 \mathrm{H}, \mathrm{s}, 2 \times \mathrm{CH}_{3}\right), 3.98\left(3 \mathrm{H}, \mathrm{s}, \mathrm{CH}_{3}\right), 7.19(2 \mathrm{H}, \mathrm{s}, \mathrm{H}-2, \mathrm{H}-6), 7.65$ $(1 \mathrm{H}, \mathrm{s}, \mathrm{CH})$.

Ethyl (E)-2-cyano-3-(2,5-dimethoxyphenyl)-2-propenoate (2c). Prepared from 2,5dimethoxybenzaldehyde 1a $(3.320 \mathrm{~g}, 20 \mathrm{mmol})$ and ethyl cyanoacetate $(2.13 \mathrm{ml}, 20 \mathrm{mmol})$ in $91 \%$ (4.750 g) yield, mp 76-78 ${ }^{\circ} \mathrm{C}$ (from ethanol). IR (KBr) cm ${ }^{-1}: 2223.3(\mathrm{CN}), 1702.1(\mathrm{CO})$. NMR data: $\delta_{\mathrm{H}}\left(\mathrm{CDCl}_{3}\right) 1.41\left(3 \mathrm{H}, \mathrm{t}, J_{\mathrm{CH}-\mathrm{CH}}=7.1 \mathrm{~Hz}, \mathrm{CH}_{3}\right), 3.83\left(3 \mathrm{H}, \mathrm{s}, \mathrm{CH}_{3}\right), 3.89\left(3 \mathrm{H}, \mathrm{s}, \mathrm{CH}_{3}\right)$, $4.38\left(2 \mathrm{H}, \mathrm{q}, J_{\mathrm{CH}-\mathrm{CH}}=7.1 \mathrm{~Hz}, \mathrm{CH}_{2}\right), 6.90\left(1 \mathrm{H}, \mathrm{d}, J_{\mathrm{H} 3-\mathrm{H} 4}=9.0 \mathrm{~Hz}, \mathrm{H}-3\right), 7.09\left(1 \mathrm{H}, \mathrm{dd}, J_{\mathrm{H} 3-\mathrm{H} 4}=9.0\right.$ $\left.\mathrm{Hz}, J_{\mathrm{H} 4-\mathrm{H} 6}=3.0 \mathrm{~Hz}, \mathrm{H}-4\right), 7.88\left(1 \mathrm{H}, \mathrm{d}, J_{\mathrm{H} 4-\mathrm{H} 6}=3.0 \mathrm{~Hz}, \mathrm{H}-6\right), 8.37(1 \mathrm{H}, \mathrm{s}, \mathrm{CH}) . \delta_{\mathrm{C}}\left(\mathrm{CDCl}_{3}\right) 14.12$, 55.80, 56.13, 62.42, 102.04, 112.10, 112.44, 115.96, 120.66, 122.30, 149.36, 153.29, 153.91, 162.74. $\mathrm{m} / \mathrm{z}(\mathrm{EI}): 261\left(\mathrm{M}^{+}\right)$. Anal. Calcd. for $\mathrm{C}_{14} \mathrm{H}_{15} \mathrm{NO}_{4}$ : C, 64.36; H, 5.79; $\mathrm{N}, 5.38$. Found: $\mathrm{C}$, 64.15; H, 5.82; N, 5.63.

\section{General procedure for preparation of 2-cyano-3-aryloxirane derivatives 3}

Solution of methylenmalononitrile derivative $2(10 \mathrm{mmol})$ in $30 \mathrm{ml}$ of acetonitrile was cooled on an ice bath to $0{ }^{\circ} \mathrm{C}$. $30 \mathrm{ml}$ of $3 \%$ solution of sodium chlorate(I) was added dropwise during period of $10 \mathrm{~min}$. $\mathrm{pH}$ of the reaction mixture was simultaneously with addition of $\mathrm{NaOCl}$ adjusted to $8-9$ using $2.5 \mathrm{M}$ solution of $\mathrm{H}_{2} \mathrm{SO}_{4}$. Reaction mixture was stirred for additional $10 \mathrm{~min}$ 
at $0{ }^{\circ} \mathrm{C} .200 \mathrm{ml}$ of ice cold water was added to reaction mixture and the product precipitated. It was collected by filtration and recrystallized from an appropriate solvent. The following compounds were prepared in this manner:

3-(2,5-Dimethoxyphenyl)-2,2-oxiranedicarbonitrile (3a). Prepared from 2-[(2,5-dimethoxyphenyl)methylen]malononitrile 2a $(2.140 \mathrm{~g}, 10 \mathrm{mmol})$ in $79 \%(1.817 \mathrm{~g})$ yield, $\mathrm{mp} 118-119{ }^{\circ} \mathrm{C}$ (from a mixture of hexane and toluene). IR $(\mathrm{KBr}) \mathrm{cm}^{-1}: 2248.4(\mathrm{CN})$. NMR data: $\delta_{\mathrm{H}}\left(\mathrm{CDCl}_{3}\right)$ $3.76\left(3 \mathrm{H}, \mathrm{s}, \mathrm{CH}_{3}\right), 3.88\left(3 \mathrm{H}, \mathrm{s}, \mathrm{CH}_{3}\right), 4.92(1 \mathrm{H}, \mathrm{s}, \mathrm{CH}), 6.89\left(1 \mathrm{H}, \mathrm{d}, J_{\mathrm{H} 4-\mathrm{H} 6}=3.0 \mathrm{~Hz}, \mathrm{H}-6\right), 6.98$ $\left(1 \mathrm{H}, \mathrm{d}, J_{\mathrm{H} 3-\mathrm{H} 4}=9.0 \mathrm{~Hz}, \mathrm{H}-3\right), 6.74\left(1 \mathrm{H}, \mathrm{dd}, J_{\mathrm{H} 3-\mathrm{H} 4}=9.0 \mathrm{~Hz}, J_{\mathrm{H} 4-\mathrm{H} 6}=3.0 \mathrm{~Hz}, \mathrm{H}-4\right) . \delta_{\mathrm{C}}\left(\mathrm{CDCl}_{3}\right)$ $41.33,55.89,56.06,62.78,110.46,111.63,111.83,111.85,117.05,117.51,152.81,153.64 . \mathrm{m} / \mathrm{z}$ (EI): $230\left(\mathrm{M}^{+}\right)$. Anal. Calcd. for $\mathrm{C}_{12} \mathrm{H}_{10} \mathrm{~N}_{2} \mathrm{O}_{3}$ : C, 62.61; H, 4.38; N, 12.17. Found: C, 62.32; H, 4.44; N, 12.09 .

Ethyl 2-cyano-3-(2,5-dimethoxyphenyl)-2-oxiranecarboxylate (3b). Prepared from ethyl (E)2-cyano-3-(2,5-dimethoxyphenyl)-2-propenoate 2c (2.610 g, $10 \mathrm{mmol})$ in $88 \%$ (2.438 g) yield, mp 69-71 ${ }^{\circ} \mathrm{C}$ (from a mixture of hexane and toluene). IR (KBr) cm ${ }^{-1}: 2250.2(\mathrm{CN}), 1750.2$ (COOEt). NMR data: $\delta_{\mathrm{H}}\left(\mathrm{CDCl}_{3}\right) 1.40\left(3 \mathrm{H}, \mathrm{t}, J_{\mathrm{CH}-\mathrm{CH}}=7.2 \mathrm{~Hz}, \mathrm{CH}_{3}\right), 3.78\left(3 \mathrm{H}, \mathrm{s}, \mathrm{CH}_{3}\right), 3.83$ $\left(3 \mathrm{H}, \mathrm{s}, \mathrm{CH}_{3}\right), 4.40\left(2 \mathrm{H}, \mathrm{q}, J_{\mathrm{CH}-\mathrm{CH}}=7.2 \mathrm{~Hz}, \mathrm{CH}_{2}\right), 4.73(1 \mathrm{H}, \mathrm{s}, \mathrm{CH}), 6.84\left(1 \mathrm{H}, \mathrm{d}, J_{\mathrm{H} 4-\mathrm{H} 6}=3.0 \mathrm{~Hz}\right.$, $\mathrm{H}-6), 6.86\left(1 \mathrm{H}, \mathrm{d}, J_{\mathrm{H} 3-\mathrm{H} 4}=9.0 \mathrm{~Hz}, \mathrm{H}-3\right), 6.74\left(1 \mathrm{H}, \mathrm{dd}, J_{\mathrm{H} 3-\mathrm{H} 4}=9.0 \mathrm{~Hz}, J_{\mathrm{H} 4-\mathrm{H} 6}=3.0 \mathrm{~Hz}, \mathrm{H}-4\right) . \delta_{\mathrm{C}}$ $\left(\mathrm{CDCl}_{3}\right)$ 13.62, 52.59, 55.41, 56.12, 60.47, 63.59, 111.70, 112.37, 113.54, 115.70, 119.50, 152.09, 152.88, 162.21. m/z (EI): $277\left(\mathrm{M}^{+}\right)$. Anal. Calcd. for $\mathrm{C}_{14} \mathrm{H}_{15} \mathrm{NO}_{5}: \mathrm{C}, 60.64 ; \mathrm{H} \mathrm{5.45;} \mathrm{N,}$ 5.05. Found: C, 60.49; H, 5.59; N, 4.82.

3-(4-Chloro-2,5-dimethoxyphenyl)-2,2-oxiranedicarbonitrile (3c). Solution of 2-[(2,5dimethoxyphenyl)methylen]malononitrile 2a $(0.935 \mathrm{~g}, 5 \mathrm{mmol})$ in $15 \mathrm{ml}$ of acetonitrile was cooled on an ice bath to $0{ }^{\circ} \mathrm{C} .15 \mathrm{ml}$ of $10 \%$ solution of sodium chlorate(I) was added dropwise during period of $10 \mathrm{~min} . \mathrm{pH}$ of the reaction mixture was simultaneously with addition of $\mathrm{NaOCl}$ adjusted to 5-6 using 2.5 M solution of $\mathrm{H}_{2} \mathrm{SO}_{4}$. Reaction mixture was stirred for additional 20 min at $0{ }^{\circ} \mathrm{C} .100 \mathrm{ml}$ of ice cold water was added to reaction mixture and the product precipitated. It was collected by filtration and recrystallized from a mixture of hexane and toluene to give compound 3c in $77 \%$ (1.016 g) yield, $\mathrm{mp} 136-138{ }^{\circ} \mathrm{C}$. IR $(\mathrm{KBr}) \mathrm{cm}^{-1}: 2253.8(\mathrm{CN})$. NMR data: $\delta_{\mathrm{H}}\left(\mathrm{CDCl}_{3}\right) 3.85\left(3 \mathrm{H}, \mathrm{s}, \mathrm{CH}_{3}\right), 3.90\left(3 \mathrm{H}, \mathrm{s}, \mathrm{CH}_{3}\right), 4.92(1 \mathrm{H}, \mathrm{s}, \mathrm{CH}), 6.75(1 \mathrm{H}, \mathrm{s}, \mathrm{H}-3), 7.03(1 \mathrm{H}$, s, H-6). $\delta_{\mathrm{C}}\left(\mathrm{CDCl}_{3}\right) 41.28,56.28,56.83,62.30,110.02,110.24,111.48,113.54,115.19,129.12$, 149.37, 152.62. $\mathrm{m} / \mathrm{z}(\mathrm{EI}): 264\left(\mathrm{M}^{+}\right)$. Anal. Calcd. for $\mathrm{C}_{12} \mathrm{H}_{9} \mathrm{~N}_{2} \mathrm{O}_{3} \mathrm{Cl}: \mathrm{C}, 54.46 ; \mathrm{H}, 3.43 ; \mathrm{N}, 10.58$. Found: C, 54.30; H, 3.48; N, 10.33 .

3-(2-Chloro-3,4,5-trimethoxyphenyl)-2,2-oxiranedicarbonitrile (3d). Solution of 2-[(3,4,5trimethoxyphenyl)methylen]malononitrile $2 \mathbf{b}(2.440 \mathrm{~g}, 10 \mathrm{mmol})$ in $30 \mathrm{ml}$ of acetonitrile was cooled on an ice bath to $0{ }^{\circ} \mathrm{C}$. $90 \mathrm{ml}$ of $3 \%$ solution of sodium chlorate(I) was added dropwise during period of $10 \mathrm{~min}$. Reaction mixture was stirred for additional $20 \mathrm{~min}$ at $0{ }^{\circ} \mathrm{C} .100 \mathrm{ml}$ of ice cold water was added to reaction mixture and the product precipitated. It was collected by filtration and recrystallized from a mixture of hexane and toluene to give compound $\mathbf{3 d}$ in $57 \%$ (1.676g) yield, mp $110-112{ }^{\circ} \mathrm{C}$. IR $(\mathrm{KBr}) \mathrm{cm}^{-1}: 2258.2(\mathrm{CN})$. NMR data: $\delta_{\mathrm{H}}\left(\mathrm{CDCl}_{3}\right) 3.86(3 \mathrm{H}, \mathrm{s}$, $\left.\mathrm{CH}_{3}\right), 3.93\left(3 \mathrm{H}, \mathrm{s}, \mathrm{CH}_{3}\right), 3.95\left(3 \mathrm{H}, \mathrm{s}, \mathrm{CH}_{3}\right), 4.95(1 \mathrm{H}, \mathrm{s}, \mathrm{CH}), 6.58(1 \mathrm{H}, \mathrm{s}, \mathrm{H}-6) . \delta_{\mathrm{C}}\left(\mathrm{CDCl}_{3}\right)$ 
42.03, 56.63, 61.23, 61.51, 63.67, 106.50, 111.43, 112.45, 119.29, 123.07, 144.70, 149.89, 152.74. $\mathrm{m} / \mathrm{z}(\mathrm{EI}): 294\left(\mathrm{M}^{+}\right)$. Anal. Calcd. for $\mathrm{C}_{13} \mathrm{H}_{11} \mathrm{~N}_{2} \mathrm{O}_{4} \mathrm{Cl}: \mathrm{C}, 52.98 ; \mathrm{H}, 3.76 ; \mathrm{N}, 9.50$. Found: C, 52.93; H, 3.85; N, 9.15.

\section{General procedure for preparation of 4,5-dihydro-1,3-thiazol-4-one derivatives 5, 7}

A mixture of 3-aryl-2,2-oxiranedicarbonitrile 3a or $3 \mathbf{d}(1 \mathrm{mmol})$ and thiourea derivative 4 $(1 \mathrm{mmol})$ was dissolved in $10 \mathrm{ml}$ of acetonitrile and stirred at room temperature for $24 \mathrm{~h}$. The product precipitated from the reaction mixture and was collected by filtration. It was recrystallized from appropriate solvent. The following compounds were prepared in this manner. 2-Amino-5-(2,5-dimethoxyphenyl)-4,5-dihydro-1,3-thiazol-4-one (5a). Prepared from 3-(2,5dimethoxyphenyl)-2,2-oxiranedicarbonitrile 3a $(0.230 \mathrm{~g}, 1 \mathrm{mmol})$ and thiourea $4 \mathbf{a}(0.076 \mathrm{~g}$, $1 \mathrm{mmol})$ in $68 \%(0.171 \mathrm{~g})$ yield, $\mathrm{mp} 196-198{ }^{\circ} \mathrm{C}$ (from ethanol). IR $(\mathrm{KBr}) \mathrm{cm}^{-1}: 3318.1\left(\mathrm{NH}_{2}\right)$, $1682.5(\mathrm{CO})$. NMR data: $\delta_{\mathrm{H}}\left(\mathrm{DMSO}_{-} \mathrm{d}_{6}\right) 3.68\left(3 \mathrm{H}, \mathrm{s}, \mathrm{CH}_{3}\right), 3.70\left(3 \mathrm{H}, \mathrm{s}, \mathrm{CH}_{3}\right), 5.34(1 \mathrm{H}, \mathrm{s}, \mathrm{CH})$, $6.73\left(1 \mathrm{H}, \mathrm{d}, J_{\mathrm{H} 4-\mathrm{H} 6}=3.0 \mathrm{~Hz}, \mathrm{H}-6\right), 6.87\left(1 \mathrm{H}, \mathrm{dd}, J_{\mathrm{H} 3-\mathrm{H} 4}=9.0 \mathrm{~Hz}, J_{\mathrm{H} 4-\mathrm{H} 6}=3.0 \mathrm{~Hz}, \mathrm{H}-4\right), 6.93(1 \mathrm{H}$, $\left.\mathrm{d}, J_{\mathrm{H} 3-\mathrm{H} 4}=9.0 \mathrm{~Hz}, \mathrm{H}-3\right), 8.73\left(1 \mathrm{H}\right.$, br s, NH), $8.99\left(1 \mathrm{H}\right.$, br s, NH). $\delta_{\mathrm{C}}\left(\mathrm{DMSO}_{\mathrm{d}}\right)$ 54.60, 55.40, 56.21, 112.72, 113.66, 115.55, 126.49, 151.34, 153.03, 181.30, 187.85. m/z (EI): $252\left(\mathrm{M}^{+}\right)$. Anal. Calcd. for $\mathrm{C}_{11} \mathrm{H}_{12} \mathrm{~N}_{2} \mathrm{O}_{3} \mathrm{~S}$ : C, 52.37; H, 4.79; N, 11.10. Found: C, 52.08; H, 4.36; N, 10.96 .

2-Amino-5-(2-chloro-3,4,5-trimethoxyphenyl)-4,5-dihydro-1,3-thiazol-4-one (5b). Prepared from 3-(2-chloro-3,4,5-trimethoxyphenyl)-2,2-oxiranedicarbonitrile 3d (0.294 g, $1 \mathrm{mmol})$ and thiourea $4 \mathrm{a}(0.076 \mathrm{~g}, 1 \mathrm{mmol})$ in $48 \%(0.151 \mathrm{~g})$ yield, $\mathrm{mp} 264-266^{\circ} \mathrm{C}$ (from ethanol). IR $(\mathrm{KBr})$ $\mathrm{cm}^{-1}: 3240.9\left(\mathrm{NH}_{2}\right), 1680.0(\mathrm{CO}) . \mathrm{NMR}$ data: $\delta_{\mathrm{H}}\left(\mathrm{DMSO}_{6}\right) 3.78\left(3 \mathrm{H}, \mathrm{s}, \mathrm{CH}_{3}\right), 3.79(3 \mathrm{H}, \mathrm{s}$, $\left.\mathrm{CH}_{3}\right), 3.80\left(3 \mathrm{H}, \mathrm{s}, \mathrm{CH}_{3}\right), 5.64(1 \mathrm{H}, \mathrm{s}, \mathrm{CH}), 6.79(1 \mathrm{H}, \mathrm{s}, \mathrm{H}-6), 8.88(1 \mathrm{H}$, br s, NH), $9.12(1 \mathrm{H}$, br s, $\mathrm{NH}) . \mathrm{m} / \mathrm{z}(\mathrm{EI}): 316\left(\mathrm{M}^{+}\right)$. Anal. Calcd. for $\mathrm{C}_{12} \mathrm{H}_{13} \mathrm{~N}_{2} \mathrm{O}_{4} \mathrm{SCl}: \mathrm{C}, 45.50 ; \mathrm{H}, 4.14 ; \mathrm{N}, 8.84$. Found: $\mathrm{C}$, 45.65; H, 4.07; N, 8.57.

2-Anilino-5-(2,5-dimethoxyphenyl)-4,5-dihydro-1,3-thiazol-4-one (5c). Prepared from 3-(2,5dimethoxyphenyl)-2,2-oxiranedicarbonitrile 3a $(0.230 \mathrm{~g}, 1 \mathrm{mmol})$ and $N$-phenylthiourea $4 \mathbf{b}$ $(0.152 \mathrm{~g}, 1 \mathrm{mmol})$ in $70 \%(0.239 \mathrm{~g})$ yield, $\mathrm{mp} 220-221{ }^{\circ} \mathrm{C}$ (from ethanol). IR $(\mathrm{KBr}) \mathrm{cm}^{-1}: 3302.1$ $(\mathrm{NH}), 1720.9(\mathrm{CO})$. NMR data: $\delta_{\mathrm{H}}\left(\mathrm{DMSO}_{6}\right) 3.72\left(3 \mathrm{H}, \mathrm{s}, \mathrm{CH}_{3}\right), 3.78\left(3 \mathrm{H}, \mathrm{s}, \mathrm{CH}_{3}\right), 5.68(1 \mathrm{H}, \mathrm{s}$, $\mathrm{CH}), 6.92\left(1 \mathrm{H}, \mathrm{dd}, J_{\mathrm{H} 3-\mathrm{H} 4}=9.0 \mathrm{~Hz}, J_{\mathrm{H} 4-\mathrm{H} 6}=3.0 \mathrm{~Hz}, \mathrm{H}-4\right), 6.99\left(1 \mathrm{H}, \mathrm{d}, J_{\mathrm{H} 4-\mathrm{H} 6}=3.0 \mathrm{~Hz}, \mathrm{H}-6\right), 7.02$ $\left(1 \mathrm{H}, \mathrm{d}, J_{\mathrm{H} 3-\mathrm{H} 4}=9.0 \mathrm{~Hz}, \mathrm{H}-3\right), 7.28-7.53(5 \mathrm{H}, \mathrm{m}, \mathrm{Ph}), 9.17\left(1 \mathrm{H}\right.$, br s, NH). $\delta_{\mathrm{C}}\left(\mathrm{DMSO}-\mathrm{d}_{6}\right) 48.99$, 55.54, 56.47, 113.16, 114.43, 116.92, 125.74, 128.28, 128.54, 128.96, 135.59, 151.20, 153.01, 157.20, 172.73. $\mathrm{m} / \mathrm{z}(\mathrm{EI}): 328\left(\mathrm{M}^{+}\right)$. Anal. Calcd. for $\mathrm{C}_{17} \mathrm{H}_{16} \mathrm{~N}_{2} \mathrm{O}_{3} \mathrm{~S}: \mathrm{C}, 62.18 ; \mathrm{H}, 4.91 ; \mathrm{N}, 8.53$. Found: C, 61.87; H, 4.72; N, 8.23.

2-Anilino-5-(2-chloro-3,4,5-trimethoxyphenyl)-4,5-dihydro-1,3-thiazol-4-one (5d). Prepared from 3-(2-chloro-3,4,5-trimethoxyphenyl)-2,2-oxiranedicarbonitrile 3d (0.294 g, $1 \mathrm{mmol}$ ) and $\mathrm{N}$ phenylthiourea $4 \mathbf{b}(0.152 \mathrm{~g}, 1 \mathrm{mmol})$ in $37 \%(0.145 \mathrm{~g})$ yield, $\mathrm{mp} 202-203{ }^{\circ} \mathrm{C}$ (from ethanol). IR $(\mathrm{KBr}) \mathrm{cm}^{-1}: 3278.9(\mathrm{NH}), 1716.2(\mathrm{CO}) . \mathrm{NMR}$ data: $\delta_{\mathrm{H}}\left(\mathrm{DMSO}_{6}\right) 3.81\left(3 \mathrm{H}, \mathrm{s}, \mathrm{CH}_{3}\right), 3.82(3 \mathrm{H}$, s, $\left.\mathrm{CH}_{3}\right), 3.84\left(3 \mathrm{H}, \mathrm{s}, \mathrm{CH}_{3}\right), 5.97(1 \mathrm{H}, \mathrm{s}, \mathrm{CH}), 7.05(1 \mathrm{H}, \mathrm{s}, \mathrm{H}-6), 7.31-7.51(5 \mathrm{H}, \mathrm{m}, \mathrm{Ph}), 9.40(1 \mathrm{H}$, br s, NH). m/z (EI): $392\left(\mathrm{M}^{+}\right)$. Anal. Calcd. for $\mathrm{C}_{18} \mathrm{H}_{17} \mathrm{~N}_{2} \mathrm{O}_{4} \mathrm{SCl}$ : C, 55.03; H, 4.36; N, 7.13. Found: C, 55.10; H, 4.70; N, 6.94. 
2-Acetylamino-5-(2,5-dimethoxyphenyl)-4,5-dihydro-1,3-thiazol-4-one (5e). Prepared from 3(2,5-dimethoxyphenyl)-2,2-oxiranedicarbonitrile 3a $(0.230 \mathrm{~g}, 1 \mathrm{mmol})$ and $N$-acetylthiourea $4 \mathbf{c}$ $(0.118 \mathrm{~g}, 1 \mathrm{mmol})$ in $57 \%(0.168 \mathrm{~g})$ yield, $\mathrm{mp} 210-211^{\circ} \mathrm{C}$ (from ethanol). IR (KBr) cm $\mathrm{cm}^{-1}: 3119.7$ $(\mathrm{NH}), 1716.7(\mathrm{CO})$. NMR data: $\delta_{\mathrm{H}}\left(\mathrm{DMSO}_{-} \mathrm{d}_{6}\right) 2.13\left(3 \mathrm{H}, \mathrm{s}, \mathrm{B}-\mathrm{CH}_{3}\right), 2.19\left(3 \mathrm{H}, \mathrm{s}, \mathrm{A}-\mathrm{CH}_{3}\right), 3.66$, $3.69\left(6 \mathrm{H}, 2 \times \mathrm{s}, 2 \times \mathrm{A}-\mathrm{CH}_{3}\right), 3.71,3.77\left(6 \mathrm{H}, 2 \times \mathrm{s}, 2 \times \mathrm{B}^{-\mathrm{CH}_{3}}\right), 5.28(1 \mathrm{H}, \mathrm{s}, \mathrm{A}-\mathrm{CH}), 6.70(1 \mathrm{H}, \mathrm{dd}$, $\left.J_{\mathrm{H} 3-\mathrm{H} 4}=9.0 \mathrm{~Hz}, J_{\mathrm{H} 4-\mathrm{H} 6}=3.0 \mathrm{~Hz}, \mathrm{~B}-\mathrm{H}-4\right), 6.83-6.97(4 \mathrm{H}, \mathrm{m}, \mathrm{A}-\mathrm{H}-3, \mathrm{~A}-\mathrm{H}-4, \mathrm{~A}-\mathrm{H}-6, \mathrm{~B}-\mathrm{H}-4), 7.67$ $\left(1 \mathrm{H}, \mathrm{d}, J_{\mathrm{H} 3-\mathrm{H} 4}=3.0 \mathrm{~Hz}, \mathrm{~B}-\mathrm{H}-6\right), 10.69(1 \mathrm{H}$, br s, B-NH), $11.79(1 \mathrm{H}$, br s, B-OH $), 12.62(1 \mathrm{H}$, br s, A-NH); ratio form $(\mathrm{A})$ : form $(\mathrm{B})=1.3: 1 . \delta_{\mathrm{C}}\left(\mathrm{DMSO}_{6}\right)$ 22.51, 23.85, 51.28, 55.20, 55.41, 56.20, 56.37, 94.18, 110.60, 112.84, 113.67, 113.93, 116.48, 122.76, 124.93, 148.10, 151.25, 153.02, 153.20, 154.91, 155.12, 168.30, 172.85, 172.90, 180.17, 187.86. m/z (EI): $294\left(\mathrm{M}^{+}\right)$. Anal. Calcd. for $\mathrm{C}_{13} \mathrm{H}_{14} \mathrm{~N}_{2} \mathrm{O}_{4} \mathrm{~S}: \mathrm{C}, 53.05 ; \mathrm{H}, 4.79 ; \mathrm{N}, 9.52$. Found: C, 52.51; H, 5.05; N, 9.14.

2-Acetylamino-5-(2,5-dimethoxyphenyl)-4,5-dihydro-1,3-thiazol-4-one (5f). Prepared from 3(2-chloro-3,4,5-trimethoxyphenyl)-2,2-oxiranedicarbonitrile $3 d(0.294 \mathrm{~g}, 1 \mathrm{mmol})$ and $\mathrm{N}$ acetylthiourea $4 \mathrm{c}(0.118 \mathrm{~g}, 1 \mathrm{mmol})$ in $36 \%(0.129 \mathrm{~g})$ yield, $\mathrm{mp} 235-236{ }^{\circ} \mathrm{C}$ (from ethanol). IR $(\mathrm{KBr}) \mathrm{cm}^{-1}: 3121.3(\mathrm{NH}), 1711.3(\mathrm{CO}) . \mathrm{NMR}$ data: $\delta_{\mathrm{H}}\left(\mathrm{DMSO}_{6}\right) 2.14\left(3 \mathrm{H}, \mathrm{s}, \mathrm{B}-\mathrm{CH}_{3}\right), 2.21$ $\left(3 \mathrm{H}, \mathrm{s}, \mathrm{A}-\mathrm{CH}_{3}\right), 3.79-3.81\left(14 \mathrm{H}, \mathrm{m}, 3 \times \mathrm{A}-\mathrm{CH}_{3}, 3 \times \mathrm{B}-\mathrm{CH}_{3}\right), 5.54(1 \mathrm{H}, \mathrm{s}, \mathrm{A}-\mathrm{CH}), 6.89$ (1H, s, AH-6), 7.05 (1H, s, B-H-6), 10.41 (1H, br s, B-NH), 1.95 (1H, br s, B-OH), 12.76 (1H, br s, A$\mathrm{NH})$; ratio form $(\mathrm{A})$ : form $(\mathrm{B})=1.7: 1 . \mathrm{m} / \mathrm{z}(\mathrm{EI}): 358\left(\mathrm{M}^{+}\right)$. Anal. Calcd. for $\mathrm{C}_{14} \mathrm{H}_{15} \mathrm{~N}_{2} \mathrm{O}_{5} \mathrm{SCl}$ : C, 46.86; H, 4.21; N, 7.81. Found: C, 47.03; H, 4.26; N, 7.47.

2-(2,5-Dimethoxyphenyl)-2,3,5,6-tetrahydroimidazo[2,1-b][1,3]thiazol-3,6-dione (7). Prepared from 3-(2,5-dimethoxyphenyl)-2,2-oxiranedicarbonitrile 3a $(0.230 \mathrm{~g}, 1 \mathrm{mmol})$ and 2-thioxo-4imidazoline $(0.100 \mathrm{~g}, 1 \mathrm{mmol})$ in $55 \%(0.160 \mathrm{~g})$ yield, $\mathrm{mp} 189-190^{\circ} \mathrm{C}$ (from ethanol). IR (KBr) $\mathrm{cm}^{-1}: 1742.4(\mathrm{CO})$. NMR data: $\delta_{\mathrm{H}}\left(\mathrm{DMSO}_{-} \mathrm{d}_{6}\right) 3.71\left(3 \mathrm{H}, \mathrm{s}, \mathrm{CH}_{3}\right), 3.72\left(3 \mathrm{H}, \mathrm{s}, \mathrm{CH}_{3}\right), 4.32(1 \mathrm{H}, \mathrm{d}$, $\left.J_{\mathrm{CH}-\mathrm{CH}}=7.0 \mathrm{~Hz}, \mathrm{CH}\right), 4.45\left(1 \mathrm{H}, \mathrm{d}, J_{\mathrm{CH}-\mathrm{CH}}=7.0 \mathrm{~Hz}, \mathrm{CH}\right), 5.94(1 \mathrm{H}, \mathrm{s}, \mathrm{CH}), 6.97\left(1 \mathrm{H}, \mathrm{dd}, J_{\mathrm{H} 3-\mathrm{H} 4}=\right.$ $\left.9.0 \mathrm{~Hz}, J_{\mathrm{H} 4-\mathrm{H} 6}=3.0 \mathrm{~Hz}, \mathrm{H}-4\right), 7.03\left(1 \mathrm{H}, \mathrm{d}, J_{\mathrm{H} 3-\mathrm{H} 4}=9.0 \mathrm{~Hz}, \mathrm{H}-3\right), 7.10\left(1 \mathrm{H}, \mathrm{d}, J_{\mathrm{H} 4-\mathrm{H} 6}=3.0 \mathrm{~Hz}, \mathrm{H}-\right.$ 6). $\delta_{\mathrm{C}}\left(\mathrm{DMSO}_{-} \mathrm{d}_{6}\right) 49.12,52.67,55.50,56.41,112.93,115.41,117.09,122.67,151.29,152.98$, 167.10, 172.73, 186.27. m/z (EI): $292\left(\mathrm{M}^{+}\right)$. Anal. Calcd. for $\mathrm{C}_{13} \mathrm{H}_{12} \mathrm{~N}_{2} \mathrm{O}_{4} \mathrm{~S}: \mathrm{C}, 53.42 ; \mathrm{H}, 4.14 ; \mathrm{N}$, 9.58. Found: C, 53.31; H, 4.15; N, 9.36.

Ethyl 2-amino-5-(2,5-dimethoxyphenyl)-1,3-thiazol-4-carboxylate (8). A mixture of ethyl 2cyano-3-(2,5-dimethoxyphenyl)-2-oxiranecarbonitrile $3 \mathbf{b}(0.277 \mathrm{~g}, 1 \mathrm{mmol})$ and thiourea $4 \mathbf{a}$ $(0.076 \mathrm{~g}, 1 \mathrm{mmol})$ was dissolved in $5 \mathrm{ml}$ of acetonitrile and refluxed for $6 \mathrm{~h}$. The solvent was evaporated under reduced pressure and an oily residue remained. After addition of a mixture of diethyl ether and ethanol a white precipitate was formed. It was collected by filtration and recrystalized from a mixture of ethyl acetate and heptane to give the product 8 in $67 \%(0.206 \mathrm{~g})$ yield, mp 130-131 ${ }^{\circ} \mathrm{C}$ (from ethanol). IR (KBr) cm ${ }^{-1}: 3454.4\left(\mathrm{NH}_{2}\right), 1722.7$ (COOEt). NMR data: $\delta_{\mathrm{H}}\left(\mathrm{DMSO}_{-} \mathrm{d}_{6}\right) 1.03\left(3 \mathrm{H}, \mathrm{t}, J_{\mathrm{CH}-\mathrm{CH}}=7.0 \mathrm{~Hz}, \mathrm{CH}_{3}\right), 3.66\left(3 \mathrm{H}, \mathrm{s}, \mathrm{CH}_{3}\right), 3.71\left(3 \mathrm{H}, \mathrm{s}, \mathrm{CH}_{3}\right)$, $4.01\left(2 \mathrm{H}, \mathrm{q}, J_{\mathrm{CH}-\mathrm{CH}}=7.0 \mathrm{~Hz}, \mathrm{CH}_{2}\right), 6.79\left(1 \mathrm{H}, \mathrm{d}, J_{\mathrm{H} 4-\mathrm{H} 6}=3.0 \mathrm{~Hz}, \mathrm{H}-6\right), 6.89\left(1 \mathrm{H}, \mathrm{dd}, J_{\mathrm{H} 3-\mathrm{H} 4}=9.0\right.$ $\left.\mathrm{Hz}, J_{\mathrm{H} 4-\mathrm{H} 6}=3.0 \mathrm{~Hz}, \mathrm{H}-4\right), 6.99\left(1 \mathrm{H}, \mathrm{d}, J_{\mathrm{H} 3-\mathrm{H} 4}=9.0 \mathrm{~Hz}, \mathrm{H}-3\right), 7.20\left(2 \mathrm{H}\right.$, br s, $\left.\mathrm{NH}_{2}\right) . \delta_{\mathrm{C}}\left(\mathrm{CDCl}_{3}\right)$ $14.10,55.75,56.13,60.91,112.58,114.46,117.18,121.37,127.41,138.00,151.00,152.94$, 
162.39, 166.82. $\mathrm{m} / \mathrm{z}(\mathrm{EI}): 308\left(\mathrm{M}^{+}\right)$. Anal. Calcd. for $\mathrm{C}_{14} \mathrm{H}_{16} \mathrm{~N}_{2} \mathrm{O}_{4} \mathrm{~S}: \mathrm{C}, 54.53 ; \mathrm{H}, 5.23 ; \mathrm{N}, 9.08$ Found: C, 54.66 H, 5.38; N, 9.41.

\section{General procedure for preparation of 5-cyano-1,3-oxathiole derivatives 9}

A mixture of 3-aryl-2,2-oxiranedicarbonitrile 3a or 3d (1 mmol) and $\mathrm{KSCN}$ (0.194 g, 2 mmol) was dissolved in $10 \mathrm{ml}$ of acetic anhydride and stirred at room temperature for $24 \mathrm{~h}$. The product precipitated from the reaction mixture and was collected by filtration. It was recrystallized from an appropriate solvent. The following compounds were prepared in this manner:

2-Acetylimino-5-cyano-4-(2,5-dimethoxyphenyl)-1,3-oxathiole (9a). Prepared from 3-(2,5dimethoxyphenyl)-2,2-oxiranedicarbonitrile 3a $(0.230 \mathrm{~g}, 1 \mathrm{mmol})$ in $38 \%(0.115 \mathrm{~g})$ yield, $\mathrm{mp}$ 183-184 ${ }^{\circ} \mathrm{C}$ (from ethanol). IR (KBr) cm ${ }^{-1}: 2222.2(\mathrm{CN}), 1657.5(\mathrm{CO})$. NMR data: $\delta_{\mathrm{H}}$ (DMSOd $) 2.32\left(3 \mathrm{H}, \mathrm{s}, \mathrm{CH}_{3}\right), 3.77\left(3 \mathrm{H}, \mathrm{s}, \mathrm{CH}_{3}\right), 3.85\left(3 \mathrm{H}, \mathrm{s}, \mathrm{CH}_{3}\right), 7.19-7.26(3 \mathrm{H}, \mathrm{m}, \mathrm{H}-3, \mathrm{H}-4, \mathrm{H}-6)$. m/z (EI): $304\left(\mathrm{M}^{+}\right)$. Anal. Calcd. for $\mathrm{C}_{14} \mathrm{H}_{12} \mathrm{~N}_{2} \mathrm{O}_{4} \mathrm{~S}: \mathrm{C}, 55.25 ; \mathrm{H}, 3.97$; N, 9.21. Found: C, 55.30; H, 3.88; N, 9.18 .

2-Acetylimino-5-cyano-4-(2-chloro-3,4,5-trimethoxypheny)-1,3-oxathiole (9b). Prepared from 3-(2-chloro-3,4,5-trimethoxyphenyl)-2,2-oxiranedicarbonitrile 3d (0.294 g, $1 \mathrm{mmol})$ in $48 \%$ (0.177 g) yield, mp 150-151 ${ }^{\circ} \mathrm{C}$ (from ethanol). IR (KBr) cm ${ }^{-1}: 2228.9(\mathrm{CN}), 1663.9(\mathrm{CO})$. NMR data: $\delta_{\mathrm{H}}\left(\mathrm{DMSO}_{-}\right) 2.35\left(3 \mathrm{H}, \mathrm{s}, \mathrm{CH}_{3}\right), 3.84\left(3 \mathrm{H}, \mathrm{s}, \mathrm{CH}_{3}\right), 3.86\left(3 \mathrm{H}, \mathrm{s}, \mathrm{CH}_{3}\right), 3.87\left(3 \mathrm{H}, \mathrm{s}, \mathrm{CH}_{3}\right)$, $7.25(1 \mathrm{H}, \mathrm{s}, \mathrm{H}-6) . \mathrm{m} / \mathrm{z}(\mathrm{EI}): 368\left(\mathrm{M}^{+}\right)$. Anal. Calcd. for $\mathrm{C}_{15} \mathrm{H}_{13} \mathrm{~N}_{2} \mathrm{O}_{5} \mathrm{SCl}: \mathrm{C}, 48.85 ; \mathrm{H}, 3.55 ; \mathrm{N}$, 7.60. Found: C, 48.61; H, 3.67; N, 7.54.

\section{General procedure for preparation of 2-[(3-chloro-1,4-naphthoquinolyl)amino]-1,3-thiazole} derivatives 11

2,3-Dichloro-1,4-naphthoquinone $(0.277 \mathrm{~g}, 1 \mathrm{mmol})$ and the 2 -aminothiazole derivative (1 mmol) were added to a solution of potassium carbonate $(0.100 \mathrm{~g}, 1 \mathrm{mmol})$ in $5 \mathrm{ml}$ of DMF. The reaction mixture was stirred at room temperature. Addition of $2 \% \mathrm{HCl}$ solution $(10 \mathrm{ml})$ caused precipitation of a crude product from the reaction mixture. It was purified by column chromatography (eluent $\mathrm{CH}_{2} \mathrm{Cl}_{2}: \mathrm{MeOH}=50: 1$ ). The following compounds were prepared in this manner:

2-[(3-Chloro-1,4-naphthoquinolyl)amino]-5-(2,5-dimethoxyphenyl)-4,5-dihydro-1,3-thiazol4-one (11a). Prepared from 2-amino-5-(2,5-dimethoxyphenyl)-4,5-dihydro-1,3-thiazol-4-one 5a $(0.252 \mathrm{~g}, 1 \mathrm{mmol}), 12$ hours of stirring in $37 \%(0.163 \mathrm{~g})$ yield, $\mathrm{mp}>250{ }^{\circ} \mathrm{C} \mathrm{IR}(\mathrm{KBr}) \mathrm{cm}^{-1}$ : $3265.0(\mathrm{NH}) 1675.0(\mathrm{CO})$. NMR data: $\delta_{\mathrm{H}}\left(\mathrm{DMSO}_{-} \mathrm{d}_{6}\right) 3.35\left(3 \mathrm{H}, \mathrm{s}, \mathrm{CH}_{3}\right), 3.70\left(3 \mathrm{H}, \mathrm{s}, \mathrm{CH}_{3}\right), 6.85-$ 7.03 (3H, m, Ph: H-3, H-4, H-6), 7.76-8.09 (4H, m, naphthoquinone: H-5, H-6, H-7, H-8). m/z (EI): $442\left(\mathrm{M}^{+}\right)$. HRMS (m/z) : $442.040550\left(\mathrm{M}^{+}\right.$, calcd. 442.039021 for $\left.\mathrm{C}_{21} \mathrm{H}_{15} \mathrm{~N}_{2} \mathrm{O}_{5} \mathrm{SCl}\right)$.

Ethyl 2-[(3-chloro-1,4-naphthoquinolyl)amino]-5-(2,5-dimethoxyphenyl)-1,3-thiazole-4-carboxylate (11b). Prepared from ethyl 2-amino-5-(2,5-dimethoxyphenyl)-1,3-thiazol-4-carboxylate $8(0.308 \mathrm{~g}, 1 \mathrm{mmol}), 2$ hours of stirring in $67 \%(0.333 \mathrm{~g})$ yield, $\mathrm{mp} 190{ }^{\circ} \mathrm{C} \mathrm{IR}(\mathrm{KBr}) \mathrm{cm}^{-1}$ : $3227.5(\mathrm{NH}), 1715.8$ (COOEt), 1679.5, $1662.2(\mathrm{CO})$. NMR data: $\delta_{\mathrm{H}}\left(\mathrm{CDCl}_{3}\right) 1.18\left(3 \mathrm{H}, \mathrm{t}, J_{\mathrm{CH}-\mathrm{CH}}\right.$ $\left.=7.2 \mathrm{~Hz}, \mathrm{CH}_{3}\right), 3.75\left(3 \mathrm{H}, \mathrm{s}, \mathrm{CH}_{3}\right), 3.79\left(3 \mathrm{H}, \mathrm{s}, \mathrm{CH}_{3}\right), 4.24\left(2 \mathrm{H}, \mathrm{q}, J_{\mathrm{CH}-\mathrm{CH}}=7.2 \mathrm{~Hz}, \mathrm{CH}_{2}\right), 6.86-$ 
$6.95(3 \mathrm{H}, \mathrm{m}, \mathrm{Ph}: \mathrm{H}-3, \mathrm{H}-4, \mathrm{H}-6), 7.75,7.78\left(2 \mathrm{H}, 2 \mathrm{ddd}, J_{\mathrm{H} 5-\mathrm{H} 6}=J_{\mathrm{H} 6-\mathrm{H} 7}=J_{\mathrm{H} 7-\mathrm{H} 8}=7.2 \mathrm{~Hz}\right.$, naphtoquinone: H-6, H-7), $8.09\left(1 \mathrm{H}\right.$, br s, NH), $8.13\left(1 \mathrm{H}, \mathrm{ddd}, J_{\mathrm{H} 5-\mathrm{H} 6}=7.5 \mathrm{~Hz}, J_{\mathrm{H} 5-\mathrm{H} 7}=1.8 \mathrm{~Hz}\right.$, $J_{\mathrm{H} 5-\mathrm{H} 8}=0.9 \mathrm{~Hz}$, naphthoquinone: $\left.\mathrm{H}-5\right), 8.20\left(1 \mathrm{H}\right.$, ddd, $J_{\mathrm{H} 7-\mathrm{H} 8}=6.9 \mathrm{~Hz}, J_{\mathrm{H} 6-\mathrm{H} 8}=1.5 \mathrm{~Hz}, J_{\mathrm{H} 5-\mathrm{H} 8}=$ $0.9 \mathrm{~Hz}$, naphthoquinone: $\mathrm{H}-8) . \mathrm{m} / \mathrm{z}(\mathrm{EI}): 498\left(\mathrm{M}^{+}\right)$. Anal. Calcd. for $\mathrm{C}_{24} \mathrm{H}_{19} \mathrm{~N}_{2} \mathrm{O}_{6} \mathrm{SCl}: \mathrm{C}, 57.77 ; \mathrm{H}$, 3.84; N, 5.61. Found: C, 57.77; H, 3.95; N, 5.92.

\section{Acknowledgments}

This research was supported by the Ministry of Education, Science and Sport of Slovenia (331102-831200, L1-2070-0795, PO-0510-0795). We thank Professor Dr. Branko Stanovnik, Faculty of Chemistry and Chemical Technology, Ljubljana, for elemental analysis, Professor Dr. Andrej Petrič, Faculty of Chemistry and Chemical Technology, Ljubljana, for ${ }^{1} \mathrm{H}$ NMR spectra, Dr. Bogdan Kralj, Institute Jožef Stefan, Ljubljana, for mass spectrometry and Professor Dr. Dominique Lorcy and Dr. Zdenko Časar, Université de Rennes 1, France, for ${ }^{13} \mathrm{C}$ NMR spectra.

\section{References}

1. Robert, A.; Baudy-Floc'h, M.; Le Grel, P.; Foucaud A. Trends in Org. Chem. 1995, 5, 37 and references cited therein.

2. Majcen-Le Maréchal A.; Pavc J.; Robert A.; Le Grel P. J. Chem. Soc., Perkin Trans. 1 1994, 2045.

3. Majcen-Le Maréchal, A.; Le Grel, P.; Robert, A.; Pavc, J. Tetrahedron 1997, 53, 13739.

4. Ferrey, M.; Robert, A.; Foucaud, A. C. R. Acad. Sci., Paris Ser. C 1973, 277, 1153.

5. Majcen-Le Maréchal, A.; Robert, A.; Leban, I. Tetrahedron 1990, 46, 453.

6. Souizi, A.; Robert, A. C. R. Acad. Sci., Paris Ser. 2 1982, 295, 571.

7. Guillemet, M.; Robert, A.; Baudy-Floc'h, M. Tetrahedron Lett. 1995, 36, 547.

8. Majcen-Le Maréchal, A.; Robert, A.; Leban, I. J. Chem. Soc., Perkin Trans. 1 1993, 351.

9. Robert, A.; Majcen-Le Maréchal, A. J. Chem. Soc., Chem. Commun. 1978, 447.

10. Majcen-Le Maréchal, A.; Le Grel, P.; Robert, A.; Biškup, J.; Ferk, V.; Toplak, R. ARKIVOC 2001, (v), 119.

11. Thompson, R. H. Naturally Occurring Quinones, 4th Edn.; Academic Press: London, 1997.

12. Rich, P.R. Faraday Discuss. Chem. Soc. 1982, 74, 349.

13. Gutierrez, P.L. Free Radic. Biol. Med. 1989, 6, 405.

14. Powis, G. Free Radic. Biol. Med. 1989, 6, 63.

15. Ikeda, N. J. Pharm. Soc. Japan 1955, 75, 1073.

16. Martin, Y. C.; Bustard, T. M.; Lynn, K.R. J. Med. Chem. 1973, 16, 1089.

17. Ter Horst, W. P.; Felix, E. L. Ind. Eng. Chem. 1943, 35, 1255.

18. Bittner, S.; Lempert, D. Synthesis 1994, 917 and references cited therein. 
19. Jones, G. In Org. React., Adams, R.; Blatt, A. H.; Boekelheide,V.; Cairns, T. L.; Cram, D. J.; House, H. O. Eds., The Knoevenagel Condensation; John Wiley \& Sons: New York, London, Sydney, 1967; Vol. 15, Chapter 2, pp 204-267.

20. Leban, I.; Lah, N. unpublished results.

21. Rosenblatt, D. H.; Broome, G. H. J. Org. Chem. 1963, 28, 1290.

22. Robert, A. C. R. Acad. Sci., Paris Ser. 2 1966, 263, 1439.

23. Toplak, R.; Lah, N.; Volmajer, J.; Leban, I.; Majcen Le Marechal, A. Acta Cryst C 2003, in press.

24. Leban, I.; Majcen-Le Maréchal, A.; Robert, A. Croat. Chem. Acta 1993, 66, 393.

25. Breslow, D.S.; Skolnik, H. Chem. Het. Comp. 1976, 21, 76.

26. Bittner, S.; Meenakshi, C.; Temtsin, G. Tetrahedron 2001, 57, 7423.

27. Temtsin, G.; Gorohovsky, S.; Bittner, S. Tetrahedron 2002, 58, 5639.

28. Kauffmann, J. Chem. Ber. 1916, 49, 1336.

29. Lee, J. Y.; Lee, H. J.; Kim, M. Y. Polymer Bull. 1997, 38, 27. 